\title{
Application and Prospect of Robotic Technology in Medical Clinic
} Haowei Ma*

University of Malaya, Kuala Lumpur 50603, Malaysia. E-mail: mhwfangzhang@mail.com

Abstract: In the process of gradually improving the level of clinical medical treatment and continuously developing industrial technology, the application of less invasive and non-invasive surgical methods in medical clinic is increasingly widespread. In the face of this situation, the inevitable trend of the development of surgery has been inclined to minimally invasive surgery. Under the background of a large number of new technologies in the clinical application of medicine, the application space of surgical minimally invasive surgery technology has become more and more extensive. The first successful laparoscopic cholecystectomy in 1987 is an important sign of the arrival of the era of minimally invasive surgery. The research and development of surgical robots based on this is a predictor of the gradual beginning of the era of surgical information processing. At this time, it will inevitably promote the qualitative improvement of surgical accuracy, and a new era of minimally invasive surgery will gradually open. At present, the latest "Da Vinci" surgical robot developed by ISRG company has been widely used in medical clinic.

Keywords: Robotic Technology; Medical Clinic; Application; Prospect

\section{Introduction}

As an important part of people's livelihood and well-being, people pay more attention to it. Medical robots, which represent the coming of the era of artificial intelligence, have gradually entered the public's attention with their deepening application in the medical field. Surgical robotics technology was developed and applied earlier in European and American countries, followed by Europe and finally Asia. It is worth noting that although China has a large-scale medical device market, from the actual situation of huge market size, about $95 \%$ of high-end devices are often imported. Therefore, the development of medical equipment in China is particularly urgent, and one of the important directions of the development of the whole industry has begun to focus more and more on the intelligent equipment, with the application of more and more new technologies on the equipment. It can be predicted that more and more systematic and intelligent medical equipment will inevitably be formed in the future, and the era of more precise medical diagnosis will gradually come, while robotic technology will become an important component of intelligent medical care.

\section{Application of surgical robot in clinic}

The first generation of Da Vinci surgical robot was born mainly in 1999, and with the completion of the first vascular surgery in the same year, Da Vinci surgical robot began to be widely used in the medical field.

Copyright (C) 2020 Haowei Ma

doi: $10.18686 /$ aem.v9i4.176

This is an open-access article distributed under the terms of the Creative Commons Attribution Non-Commercial License

(http://creativecommons.org/licenses/by-nc/4.0/), which permits unrestricted non-commercial use, distribution, and reproduction in any medium, provided the original work is properly cited. 
Today, Da Vinci surgical robotic system has gradually been popularized in many disciplines worldwide. And the clinical application has gradually expanded to all levels and branches of surgery, through the application of surgical robots can carry out a wide range of complex surgical procedures, such as colon and hemicolectomy and other eight types of gastrointestinal surgery (gastrointestinal surgery); the first generation of Da Vinci surgical robot was born mainly in 1999, and with the completion of the first vascular surgery in the same year, Da Vinci surgical robot began to be widely used in the medical field. Today, Da Vinci surgical robotic system has gradually been popularized in many disciplines worldwide. And the clinical application has gradually expanded to all levels and branches of surgery, through the application of surgical robots can carry out a wide range of complex surgical procedures, such as colon and hemicolectomy and other eight types of gastrointestinal surgery (gastrointestinal surgery); the first operation carried out by urology with the aid of surgical robots was prostatectomy, followed by pyeloplasty and kidney transplantation. The earliest surgical field of Da Vinci surgical robots was coronary artery bypass grafting cardiac surgery, and with the success of this surgical case in 1999 , the history of surgical robots opened. It has promoted the wide application of surgical robots in various types of open-vision cardiac surgery, and Da Vinci surgical robots have also gradually been involved in a large number of thoracic and gynecological operations ${ }^{[1]}$. The wide variety of surgical procedures, the improvement of surgical accuracy, and the emergence of a large number of successful surgical cases have made surgeons increasingly recognized and respected Da Vinci surgical robots. Since the introduction of the first generation of Da Vinci surgical robots in China in 2006, surgical robots have been gradually popularized in a large number of surgical fields in the mainland of China.

Surgical robots relying on broadband technology can be docked to devices with digital interfaces, thus achieving the purpose of remote transmission and control, providing sufficient guarantee for the development of remote surgery. The successful completion of laparoscopic cholecystectomy by a long-distance manipulated surgical robot in the United States in 2001 marked a leap forward in the era of surgery. At the same time, this operation is also an effective extension of the surgical robot to the field difficult to reach by conventional laparoscopic surgery. Compared with ordinary minimally invasive surgery, the advantages of surgical robots are very significant $^{[2]}$.

\section{Other applications of robotics in medicine}

Robotic technology is not only applied in surgical operations, but also widely used in the field of rehabilitation, such as the advantages of rehabilitation medical robots in the fields of disability assistance and elderly care are also very significant. At this time, rehabilitation robots (patients with arm disability), intelligent wheelchairs (patients with lower limb disability), mobile rehabilitation robots (patients with binocular blindness) are the main areas of robot technology research. Hospital service robots mainly provide assistant functions for the completion of some work of nurses, such as food and drug delivery; while robot walking design based on sensor and motion planning algorithm is more applicable in structured environment ${ }^{[3]}$. Under the background of the gradual development of micro-electromechanical system technology, the micro-medical robotic endoscopy technology has been further studied and developed, and this technology has also begun to gradually spread to the clinic. With the increasing maturity and development of robotic technology, robotic medical assistant technology has begun to be more widely used, such as the use of medical robots in liver and kidney puncture to assist, can effectively avoid rib vessels and abdominal cavity and other important organs, thereby achieving a safer abdominal puncture.

Relevant reports show that China's micro medical robots have been successfully developed by the relevant specialties of the School of Engineering, the Chinese University of Hong Kong, and the research and development of this medical robot provides great convenience for the precise delivery of drugs to specific parts of the human body, thus implying that this technology can be applied in the future in the targeted treatment of cancer, cerebral infarction and stroke and other diseases. The micro-robot controlled by an external magnetic field can precisely deliver drugs to tiny parts of the human body. At the same time, it can shuttle in blood vessel, eye and brain, and then directly deliver drugs to places that 
are difficult to reach by traditional methods, making full breakthroughs in the limitations of traditional treatment methods that can only be passively administered by means of blood vessel in the past, so as to ensure the real realization of active targeted drug delivery.

The research of ultrasonic diagnosis robot technology is also very extensive. The related exhibition of Waseda University was held in 2013, which enabled the ultrasonic remote diagnosis robot to be operated at portable terminals such as smartphones to be displayed. In the application of this device, the main portable terminal control device is the smartphone. Later, based on the application of proprietary operating probes, the rotation control objectives of 360 and 45 degrees tilt can be well achieved. At the same time, the control of translation within $10 \mathrm{~cm}$ can also be realized to ensure that the doctor can achieve the goal of remote operation of the ultrasonic diagnostic device, which is very helpful for the doctor to understand the bleeding and other conditions in the patients during the emergency transportation process, and also can provide great help for the rapid adoption of coping strategies after the patients arrive at the hospi$\mathrm{tal}^{[4]}$.

\section{Analysis of technical defects of medical robot}

Robot technology applied in the medical field has obvious advantages such as operation precision and stability. Affected by this factor, it provides a great impetus for the development of modern medical technology. However, it is worth noting that surgical robot technology is gradually developing, so it is difficult to fully drive the traditional surgical methods, but also in medical behavior can not completely replace the role played by human beings. From the technical aspects of analysis, although robotic technology has advanced, but it still has shortcomings, specifically reflected in the following aspects.

Surgical risk. In the process of using surgical robot technology, it not only has the risk of conventional surgery, but also has a greater probability of mechanical failure such as machine crash during the operation compared with the endoscopic surgery system. It also increases the risk of intraoperative accidents while prolonging the operation time. The most important concern of patients and doctors is the risk of surgery. Once the probability of surgical risk is increased, the widespread popularity of surgical robots will be greatly restricted ${ }^{[5]}$.

Tactile feedback system. In the absence of force tactile feedback system, the surgeon's "hand feeling" will be lost, which will lead to the increasing uncertainty and risk of surgery, and the further development of surgical robots will be limited.

Operative time. From the practical application of surgical robots, although it has a high accuracy, it is worth noting that the preoperative preparation and intraoperative device replacement operations using the surgical robotic process often take a long time, and the existence of this factor will also restrict the wide application of surgical robots in clinical practice ${ }^{[6]}$.

High costs. When purchasing surgical robots, it has a higher purchase cost, while in clinical application, it will also produce a higher operation cost. In addition, affected by higher maintenance costs, it will not only make the operation of surgical robots more difficult gradually. At the same time, it is difficult to ensure that patients report for acceptance and other attitudes towards this surgical approach, which will also restrict the popularity of surgical robots.

\section{Prospects}

Surgical accuracy and reliability are significant advantages of robotic technology, so the development trend in the medical field is ideal, and good clinical effects are becoming more prominent ${ }^{[7]}$. In the future, it is still necessary to continuously improve the surgical robot technology, which can be mainly from the following aspects: strengthen the stability of the surgical system, so that the probability of mechanical failure can be effectively reduced, to provide help for the reduction of surgical risk, so that the psychological disorders of patients and operators gradually disappear; the tactile feedback system has been actively developed, which gradually reduces the uncertainty and risk of surgery. The technical research has been strengthened to ensure the flexible application of robotic technology and provide favorable conditions for the reduction of operation time. Strengthen efforts on new surgical robots to ensure that their use costs are gradually reduced while achieving more practical research and development goals of surgical robots; 
develop medical robots with a wide range of roles to ensure that robotic technology can be widely used in all aspects of the medical field ${ }^{[8]}$.

\section{Conclusion}

From the practical application of robotic technology, robotic technology has been widely promoted in the conventional application and clinical medicine of developed countries, and from the analysis of our current application situation, it is still dominated by high-end medical attributes. Under the background of further development of robotic technology, with the increasing progress and maturity of technology, it is bound to deeply and extensively study and apply various medical robotic technologies, medical robot assistant technologies, etc. At the same time, the public and patients will also recognize and accept robotic technology, and it can be seen that future robotic technology has a very broad development prospects.

\section{References}

1. Liu L, Wang D. Application and existing problems of artificial intelligence in medical field. Soft Science of Health 2020; 34(10): 23-27.

2. Tian B, Lu H, Zhang J, et al. Application of telemedicine robot in follow-up after liver transplantation from donation after cardiac death. Organ Transplantation 2019; 10(1): 79-83.

3. Hai H. Overview of research status and future development trend of dental medical robot. The Department of Oral Medicine Electronic Magazine (Electronic Edition) 2018; 5(34): 18-19.

4. Cai S, Song Y, Cao X, et al. Application status and research progress of orthopedic robots (in Chinese). Shandong Medical Journal 2018; 58(44): 90-93.

5. Guo C. Development and application of robots in craniomaxillofacial surgery remains a long and arduous task. Chinese Journal of Stomatology 2018; 53(8): 505-507.

6. Chen Z. AI for medical enabling smart medical market will reach 20 billion (in Chinese). Health Care Today 2018; (2): 4-6.

7. Zheng H. Medical robot: The curtain of the 100 billion-dollar market is opening (in Chinese). Romantic Generation 2017; (24): 18-19.

8. Zhang J, Gu H, Sun J, et al. Application and prospect of robot technology in medical clinic. Machine Tool \& Hydraulics 2016; 44(3): 52-55. 